\title{
Componentes genéticos de médias e depressão por endogamia em populações de milho-pipoca
}

\author{
Average genetic components and inbreeding depression in popcorn populations
}

\author{
Carlos Alberto Scapim ${ }^{1}$ Alessandro de Lucca e Braccini ${ }^{1}$ Ronald José Barth Pinto ${ }^{1}$ \\ Antônio Teixeira do Amaral Júnior ${ }^{2}$ Marcos de Araújo Rodovalho ${ }^{3}$ \\ Rodrigo Martins da Silva ${ }^{3}$ Lia Mara Moterle ${ }^{3}$
}

\section{RESUMO}

Dez populações de milho-pipoca (PR 038, PR 079, RR 046, SC 016, PR 017, BRS ANGELA, SC 002, PR 009, PR 023 e SE 013) foram avaliadas com os objetivos de obter estimativas de depressão por endogamia e componentes genéticos de média. O delineamento experimental utilizado foi o de blocos ao acaso em esquema de parcelas subdivididas, nos quais os tratamentos primários foram os níveis de endogamia $\left(S_{1}\right.$ e $\left.S_{0}\right)$ e os tratamentos secundários as populações, com três repetições. $O$ ensaio foi conduzido nas cidades de Maringá e Iguatemi-PR, no ano agrícola de 2001/ 2002. Foram avaliadas as características altura de plantas, altura de espigas, rendimento de grãos e capacidade de expansão. Foram observados menores valores de depressão por endogamia e predominância de efeitos gênicos aditivos para capacidade de expansão em relação ao rendimento de grãos. As populações BRS ANGELA e SC 002 apresentaram maior probabilidade de sucesso na obtenção de linhagens com boa capacidade de expansão.

Palavras-chave: Zea mays, linhagens, rendimento, capacidade de expansão.

\section{ABSTRACT}

Ten popcorn populations (PR 038, PR 079, RR 046, SC 016, PR 017, BRS ANGELA, SC 002, PR 009, PR 023 and $S E$ 013) were evaluated to estimate inbreeding depression and average genetic components. The experiment was a split plot in a randomized complete block design with three replications. The plots were the inbreeding levels $\left(S_{1}\right.$ and $\left.S_{0}\right)$ and the sub-plots were the populations in two locations (Maringá and Iguatemi-PR) during the 2001/2002 grown season. Plant and ear height, grain yield and popping expansion were evaluated. It was observed lower values of inbreeding depression and predominance of additive effects for popping expansion in relation to yield. The sucess of getting high popping expansion inbred lines is higher with BRS ANGELA and SC 002 populations.

Key words: Zea mays, inbred, yield, popping expansion.

\section{INTRODUÇÃO}

O mercado nacional de milho-pipoca tem passado por várias mudanças nos últimos cinco anos, principalmente, no que diz respeito ao sistema de parceria entre produtores e empacotadoras e a importação do produto. Até o início do ano agrícola 2003/04, o consumo nacional de milho-pipoca estava em torno de 80 mil toneladas, sendo que $75 \%$ deste mercado correspondia a milho- pipoca americano, importado principalmente da Argentina. As principais razões dessa dependência estavam relacionadas à baixa qualidade da pipoca disponível no mercado brasileiro devido à limitação de cultivares de alta qualidade e tecnologia de produção inadequada (GALVÃO et al., 2000). Na safra 2004/05, segundo informações das empresas empacotadoras, houve inversão no panorama nacional, com a entrada do híbrido simples modificado IAC-112 e a importação brasileira foi de 20 mil toneladas. Dessa forma, o País começou a diminuir a dependência de sementes importadas.

${ }^{1}$ Departamento de Agronomia, Universidade Estadual de Maringá (UEM), Av. Colombo, 5790, 87020-900, Maringá, Paraná, Brasil. E-mail: cascapim@uem.br. Autor para correspondência.

${ }^{2}$ Departamento de Agronomia, Universidade Estadual do Norte Fluminense, Rio de Janeiro, Brasil.

${ }^{3}$ Curso de Agronomia, UEM, Maringá, Paraná, Brasil. 
Apesar desses avanços, o número de cultivares é ainda bastante reduzido. O valor comercial do milho-pipoca é maior que o milho normal, o que justifica, inclusive, a aplicação de recursos federais nos programas de melhoramento vegetal (SAWAZAKI, 2001). Dessa forma, os programas de melhoramento que desenvolvem variedades e híbridos devem ser incentivados. Um dos objetivos do programa de melhoramento genético de milho-pipoca, a longo prazo, é a exploração do vigor híbrido. O método frequientemente utilizado para obtenção de linhagens homozigóticas é a autofecundação artificial, tendo como conseqüência principal a depressão por endogamia. $\mathrm{O}$ valor de uma população como fonte de linhagens é altamente dependente da depressão por endogamia em relação às várias características, principalmente rendimento de grãos, o que limita a obtenção de linhagens vigorosas para a posterior obtenção de híbridos (LIMA et al., 1984). Quanto à obtenção de linhagens com probabilidade aceitável, deve-se partir de populações que apresentem alta frequência de alelos favoráveis em relação aos diversos caracteres de interesse e baixa frequência de alelos desfavoráveis (VENCOVSKY \& BARRIGA, 1992).

Existem trabalhos em relação às estimativas de depressão por endogamia para várias características empregando diferentes populações em milho com endosperma comum (HALLAUER \& SEARS, 1973; GOOD \& HALLAUER, 1977; VIANA et al., 1982; LIMA et al., 1984; NASS \& MIRANDA FILHO, 1995 e VASAL et al., 1995). No entanto, em milho-pipoca de grãos amarelos, foi possível observar apenas duas referências nessa linha de trabalho (BRUNSON, 1937 e SIMON et al., 2004). SIMON et al. (2004) avaliaram oito populações de milho-pipoca de grãos amarelos e concluíram que a probabilidade de sucesso na obtenção de linhagens vigorosas e de alta capacidade de expansão era baixa para todas as populações. No Brasil, existem muitas populações de milho-pipoca que ainda não foram avaliadas em relação à depressão por endogamia e que poderiam ser utilizadas em programas de melhoramento. Com base no exposto, os objetivos deste trabalho foi obter estimativas da depressão por endogamia e componentes genéticos de média em dez populações de milho-pipoca.

\section{MATERIAL E MÉTODOS}

Foram utilizadas dez populações de milhopipoca, sendo oito de grãos brancos (PR 038, PR 079, RR 046, SC 016, PR 017, BRS ANGELA, SC 002 e PR 009) e duas de grãos amarelos (PR 023, SE 013). A população BRS ANGELA pertence a
EMBRAPA-Milho e Sorgo - Sete Lagoas-MG e as outras populações são derivadas de híbridos americanos e argentinos. Em relação aos métodos de melhoramento, BRS-ANGELA foi lançada, comercialmente, em 2000, após o sexto ciclo de seleção (PACHECO et al., 2001). As outras populações foram pouco melhoradas, ou seja, foram feitas apenas seleção massal para as doenças foliares. Em um campo de polinização, foram autofecundadas, aproximadamente, 250 plantas para obtenção da geração $S_{1}$; em outro campo, foi efetuada a multiplicação utilizando bulk de pólen, para obtenção da geração $S_{0}$. $O$ ensaio foi conduzido nas cidades de Maringá e Iguatemi-PR, no ano agrícola de 2001/02

O delineamento experimental foi o de blocos ao acaso em esquema de parcelas subdivididas, em que os níveis de endogamia $\left(\mathrm{S}_{1}\right.$ e $\left.\mathrm{S}_{0}\right)$ foram alocados nas parcelas e as populações nas subparcelas, com três repetições. Cada subparcela foi composta por quatro fileiras de 5,0m de comprimento espaçadas de $1,0 \mathrm{~m}$ e cinco plantas por metro, após o desbaste, correspondendo a $20 \mathrm{~m}^{2}$ de área ocupada pela subparcela e $160 \mathrm{~m}^{2}$ pela parcela. Aárea útil foi composta pelas duas fileiras centrais de cada subparcela, comportando 50 plantas. Na adubação de semeadura, utilizou-se o formulado 4-14-8, na dose de $350 \mathrm{~kg} \mathrm{ha}^{-1}$. $\mathrm{Na}$ adubação de cobertura, foram utilizados sulfato de amônio na dose de $150 \mathrm{~kg} \mathrm{ha}^{-1}$ e cloreto de potássio na dose de $50 \mathrm{~kg} \mathrm{ha}^{-1}$, dividida em duas etapas, sendo a primeira, aos 25 dias após a emergência e a segunda, quando as plantas apresentavam oito a dez folhas totalmente expandidas. Para o controle de lagarta do cartucho (Spodoptera frugiperda), utilizou-se Deltamethrine, na dosagem recomendada pelo fabricante. O controle de ervas daninhas foi feito com Atrazine+Metolachlor.

As características avaliadas foram altura de plantas (m), altura das espigas (m), rendimento de grãos $\left(\mathrm{kg} \mathrm{ha}^{-1}\right)$ e capacidade de expansão $\left(\mathrm{mL} \mathrm{g}^{-1}\right)$. Os dados referentes ao rendimento de grãos foram corrigidos para a umidade padrão (14,5\%), base úmida. A capacidade de expansão foi obtida a partir da razão entre o volume de pipoca expandida, medida numa proveta com capacidade de $1.000 \mathrm{~mL}$ e a massa de $30 \mathrm{~g}$ de grãos, medido em balança de precisão. Foram avaliadas duas amostras por parcela. A massa de grãos submetidos ao estouro foi obtida do total de grãos colhidos da parte centro-basal das espigas de cada subparcela. Antes da avaliação da capacidade de expansão, as amostras de grãos e a amostra piloto de $1,0 \mathrm{~kg}$ que serviu para monitoramento da umidade, foram armazenadas em câmara seca e fria. A avaliação da capacidade de expansão só foi efetuada, quando a umidade da amostra 
piloto atingiu uma faixa ideal de $13 \%$ a $15 \%$. Na obtenção da capacidade de expansão, foi utilizado pipocador desenvolvido pela Embrapa - Centro Nacional de Pesquisa e Desenvolvimento de Instrumentação Agropecuária (CNPDIA), baseado no testador oficial da capacidade de expansão da Cretors $\mathrm{CO}$, contendo resistência elétrica e termostato. A temperatura ideal de funcionamento deste equipamento é de $280^{\circ} \mathrm{C}$, com tempo de dois minutos e quinze segundos das amostras de grãos na panela.

Para a análise de variância conjunta, foi adotado modelo misto, no qual foram considerados aleatórios todos os efeitos, com exceção da média, níveis de endogamia, populações e da interação entre níveis de endogamia e populações. Aplicou-se o teste de Bartlett que tem distribuição de $\chi^{2}(\mathrm{P}<0,05)$ para verificar as homogeneidades de variâncias, para possibilitar a análise de variância conjunta dos dois locais (GOMEZ \& GOMEZ, 1984). Empregou-se a metodologia de GARDNER (1965) para obtenção das estimativas dos componentes genéticos de média nas populações de polinização aberta descrita da seguinte forma:

A notação proposta por GARDNER (1965), com base no modelo aditivo dominante, nas médias de uma população panmítica, indica que $\mu$ é a média geral, $\alpha$ fornece informação acerca da contribuição cumulativa dos locos homozigóticos à média da população $\delta$ representa a contribuição dos locos em heterozigose. As estimativas de médias esperadas das linhagens obtidas ao acaso na população, segundo dedução, foi: $\quad$ A estimativa da contribuição dos locos em heterozigose $(\delta)$ para uma característica, foi: . A estimativa da depressão por endogamia (DP), em porcentagem, nas populações de polinização livre foi obtida utilizando-se da fórmula seguinte:

$D P=\left(\overline{S_{0}}-\overline{S_{1}}\right) / \overline{S_{0}} * 100$ em que $\bar{S}_{0}=$ média da população original e $\bar{S}_{1}=$ média da população após uma geração de autofecundação.

\section{RESULTADOS E DISCUSSÃO}

Os resultados da análise de variância revelaram diferenças significativas $(\mathrm{P}<0,05)$ somente para a interação níveis de endogamia $\mathrm{x}$ populações, evidenciando que a intensidade da depressão por endogamia não se comportou similarmente entre as populações e que essa intensidade foi consistente nos dois locais, permitindo, dessa forma, a análise da depressão por endogamia com base nas médias das características em Maringá e Iguatemi-PR. Os coeficientes de variação de todas as características avaliadas estão dentro dos limites aceitáveis para experimentação agrícola, conforme a proposta elaborada por SCAPIM et al. (1995) (Tabela 1).

De modo geral, as estimativas da depressão por endogamia, quanto à altura de plantas e espigas foram baixas quando comparadas com rendimento de grãos (Tabela 1). Valores baixos de depressão por endogamia para estas características também foram obtidos em populações de milho normal e de milhopipoca de grãos amarelos (LIMA et al., 1984; SIMON et al., 2004). As possíveis razões das diferenças entre as estimativas de depressão por endogamia para os diversos caracteres pode ser discutida em função da redução de $50 \%$ do nível de heterozigose. A mais rápida obtenção de estabilidade de médias para altura de plantas e espigas pode ser devido a menor complexidade genética deles em relação ao caráter rendimento de grãos (JONES, 1939).

Do ponto de vista dos efeitos genéticos, as estimativas da depressão por endogamia são menores para altura da planta e espiga em relação ao rendimento de grãos porque os efeitos gênicos de dominância são menos importantes (LIMA et al., 1984). A contribuição dos locos em heterozigose (desvios devidos à dominância) às médias do rendimento de grãos das populações foi, geralmente, maior que a dos locos em homozigose (efeitos aditivos). Esse resultado implica, indiretamente, na existência de heterogeneidade genética nas populações. Em relação à altura de plantas e espigas a contribuição dos locos em homozigose (efeito aditivo) foi maior do que a contribuição dos locos em heterozigose. Resultados semelhantes foram obtidos em milho normal e em milho-pipoca de grãos amarelos (HALLAUER \& MIRANDA FILHO, 1981; SIMON et al., 2004).

As populações que apresentaram maiores rendimento de grãos, na média dos dois locais, foram PR 023 (4900 $\left.\mathrm{kg} \mathrm{ha}^{-1}\right)$, BRS-ANGELA (4500 kg ha-1), SC 016 (4300kg ha ${ }^{-1}$ ) e PR 017 (4200kg ha $\left.{ }^{-1}\right)$. Os valores encontrados são superiores às melhores médias encontradas no Ensaio Nacional de milho-pipoca, anos agrícolas 1991/92 e 1992/93 (VENDRUSCULO, 1997). Esses híbridos apresentam o mesmo potencial de rendimento de grãos do que o híbrido simples modificado IAC-112 (GALVÃO et al., 2000; SAWAZAKI et al., 2000) e são superiores ao híbrido triplo Zélia, relatado por SCAPIM et al., (2002). Essa comparação deve ser vista com cautela, em virtude de não terem sido realizados experimentos com todas as populações supracitadas, nos mesmos locais e condições climáticas, para estimar a interação populações por ambientes. A população PR 017, apesar de produtiva, tem altura de plantas na média de 2,50m, 
Tabela 1 - Altura de plantas (AP), altura de espigas (AE), rendimento de grãos (RG) e capacidade de expansão (CE) das populações parentais $\left(\mathrm{S}_{0}\right)$ e das linhagens $\left(\mathrm{S}_{1}\right)$, depressão por endogamia (DP) $(\%)$ e as estimativas de médias esperadas num conjunto de linhagens puras $(\mu+\alpha)$ e os componentes dessas características esperadas em virtude dos locos em heterozigose $(\delta)$, na média de dois locais, Iguatemi e Maringá (PR)

\begin{tabular}{|c|c|c|c|c|c|c|c|c|c|c|c|c|c|c|c|c|c|c|c|c|}
\hline \multirow[t]{2}{*}{ População } & \multicolumn{5}{|c|}{ Altura de plantas (m) } & \multicolumn{5}{|c|}{ Altura de espigas (m) } & \multicolumn{5}{|c|}{$\begin{array}{l}\text { Rendimento de grãos } \\
\qquad\left(\mathrm{kg} \mathrm{ha}^{-1}\right)\end{array}$} & \multicolumn{5}{|c|}{$\begin{array}{l}\text { Capacidade de expansão } \\
\qquad\left(\mathrm{mL} \mathrm{g}^{-1}\right)\end{array}$} \\
\hline & $\mathrm{S}_{0}$ & $\mathrm{~S}_{1}$ & DP & $\mu+\alpha$ & $\delta$ & $\mathrm{S}_{0}$ & $\mathrm{~S}_{1}$ & DP & $\mu+\alpha$ & $\delta$ & $\mathrm{S}_{0}$ & $\mathrm{~S}_{1}$ & DP & $\mu+\alpha$ & $\delta$ & $\mathrm{S}_{0}$ & $\mathrm{~S}_{1}$ & DP & $\mu+\alpha$ & $\delta$ \\
\hline PR 038 & 2,00 & 1,90 & 5,0 & 1,8 & 0,20 & 1,45 & 1,40 & 3,4 & 1,35 & 0,10 & 3000 & 2400 & 20,0 & 1800 & 1200 & 10,0 & 9,0 & 10,0 & 8,0 & 2,0 \\
\hline PR 079 & 2,30 & 2,10 & 8,7 & 1,9 & 0,40 & 1,43 & 1,28 & 10,5 & 1,13 & 0,30 & 2500 & 1900 & 24,0 & 1300 & 1200 & 15,0 & 13,0 & 13,3 & 11,0 & 4,0 \\
\hline RR 046 & 2,50 & 2,20 & 12 & 1,9 & 0,60 & 1,58 & 1,40 & 11,4 & 1,22 & 0,36 & 2740 & 1500 & 45,2 & 260 & 2480 & 6,7 & 6,0 & 10,4 & 5,3 & 1,4 \\
\hline SC 016 & 2,10 & 1,90 & 9,5 & 1,7 & 0,40 & 1,45 & 1,34 & 7,6 & 1,23 & 0,22 & 4300 & 2630 & 38,8 & 960 & 3340 & 24,0 & 21,0 & 12,5 & 18,0 & 6,0 \\
\hline PR 017 & 2,50 & 2,40 & 4,0 & 2,3 & 0,20 & 1,68 & 1,50 & 10,7 & 1,32 & 0,36 & 4200 & 2500 & 40,4 & 800 & 3400 & 19,5 & 18,0 & 7,7 & 16,5 & 3,0 \\
\hline BRS Ângela & 2,44 & 2,29 & 6,2 & 2,2 & 0,30 & 1,65 & 1,40 & 15,2 & 1,15 & 0,50 & 4500 & 3400 & 24,5 & 2300 & 2200 & 31,0 & 28,0 & 9,7 & 25,0 & 6,0 \\
\hline SC 002 & 2,20 & 2,10 & 4,5 & 2,0 & 0,20 & 1,42 & 1,32 & 7,0 & 1,22 & 0,20 & 2600 & 1900 & 27,0 & 1200 & 1400 & 27,0 & 24,0 & 11,2 & 21,0 & 6,0 \\
\hline PR 009 & 2,30 & 2,20 & 4,3 & 2,1 & 0,20 & 1,65 & 1,62 & 1,82 & 1,59 & 0,06 & 1900 & 1700 & 10,5 & 1500 & 400 & 28,0 & 26,0 & 7,2 & 24,0 & 4,0 \\
\hline PR 023 & 2,60 & 2,40 & 7,7 & 2,2 & 0,40 & 1,70 & 1,65 & 2,94 & 1,60 & 0,10 & 4900 & 3000 & 38,7 & 1100 & 3800 & 19,0 & 17,0 & 10,5 & 15,0 & 4,0 \\
\hline SE 013 & 2,38 & 2,30 & 3,4 & 2,2 & 0,16 & 1,60 & 1,50 & 6,25 & 1,40 & 0,20 & 3600 & 2700 & 25,0 & 1800 & 1800 & 17,5 & 15,0 & 14,3 & 12,5 & 5,0 \\
\hline
\end{tabular}

AP-CV(\%): erro (a): 4,05; erro (b): 6,87;

$\operatorname{AE}-\mathrm{CV}(\%)$ : erro (a): 8,13 ; erro (b): 7,35;

RG-CV(\%): erro (a): 19,0; erro (b): 18,65 ;

CE-CV(\%): erro (a): 5,20; erro (b): 5,76

não apresentando tolerância ao acamamento. As populações que apresentaram maiores valores médios de CE ( $\left.\mathrm{mLg}^{-1}\right)$, foram BRS-ANGELA (31), PR 009 (28) e SC 002 (27). A população PR 009 é susceptível a Phaeosphaeria maydis. A população SC 002 é mais tolerante a essa doença, ao acamamento e quebramento. Com essas características, a população SC 002 apresenta melhor perspectiva de sucesso, por exemplo, para o melhoramento intrapopulacional.

A depressão por endogamia em relação ao rendimento de grãos apresentou variação de $10,5 \%$ (PR 009) a 45,2\% (RR 046). Essas estimativas, com raras exceções, estão próximas ou são superiores as relatadas na cultura do milho normal (HALLAUER \& MIRANDA FILHO, 1981). Em relação ao milho-pipoca de grãos amarelos essas estimativas foram, de um modo geral, inferiores (SIMON et al., 2004). Esses autores encontraram oito estimativas de depressão por endogamia acima de $45 \%$ nas populações avaliadas em dois anos. A população RR 046 é bastante susceptível ao acamamento e a Phaeosphaeria maydis, o que dificulta a sua utilização em um programa de melhoramento.

A depressão por endogamia é maior em variedades de milho-pipoca em relação ao milho com endosperma normal, o que dificulta a obtenção de linhagens (ZINSLY \& MACHADO, 1987). Nesse contexto, isso é verdade em variedades locais de alta variabilidade genética (SAWAZAKI, 1995). As populações RR 046 (DP=45,2\%), SC 016 (DP=38,8\%), PR 017 (DP=40,4\%) e PR 023 ( DP=38,7\%) confirmam os relatos desse autor, com as mais altas estimativas de depressão por endogamia. Tratando-se de variedades melhoradas ou sintéticos, isso não deve ocorrer. A população BRS ANGELA pode ser considerada uma variedade melhorada e apresenta menor estimativa de depressão por endogamia (DP=24,5\%). Nesse aspecto, BRUNSON (1937) relata que a perda de vigor causada pela endogamia foi menor em milho-pipoca do que em milho normal.

As estimativas de depressão por endogamia em relação à capacidade de expansão variaram entre 7,2\% (PR 009) a 14,3\% (SE 013). A variação e a magnitude nos valores de depressão por endogamia para esta característica são bem inferiores aos encontrados em relação ao rendimento de grãos, o que é explicado, pela menor complexidade genética do caráter e pela predominância de efeitos gênicos aditivos. Observase que a contribuição dos locos em homozigose (efeitos aditivos) é maior do que a contribuição dos locos em heterozigose (desvios devidos à dominância). Essas estimativas estão próximas das populações de grãos amarelos, que variaram de 0,0 a $21,0 \%$, sendo dez estimativas acima de 6,0\% (SIMON et al., 2004). Vários trabalhos têm demonstrado a predominância de efeitos genéticos aditivos quanto à capacidade de expansão, confirmando boas condições de seleção para essa característica (DOFING et al., 1991; PACHECO et al., 
1998; LARISH \& BREWBAKER, 1999; PEREIRA \& AMARAL JÚNIOR, 2001; SCAPIM et al., 2002; SIMON et al., 2004). O método de seleção massal pode proporcionar progresso significativo para esse caráter (SAWAZAKI, 1995). Um aspecto positivo observado é que não houve valores negativos nas estimativas de médias esperadas em nenhuma característica. Desse modo não há superestimação dos efeitos da endogamia que poderiam levar à obtenção de estimativas irreais (negativas) das características nas linhagens. Dessa forma, a estimativa de $\mu+\alpha$ é de valor para o melhorista (VENCOVSKY \& BARRIGA, 1992).

Uma seleção de linhagens vigorosas pressupõe a existência de dispersão ou variação genética entre linhagens. Tal dispersão é causada por diferentes tipos de componentes genéticos da variação genotípica total, presente na população de origem. Pelo que foi exposto, não é tarefa fácil descrever o perfil completo de uma população, quanto à natureza da variabilidade genética, para aquilatar o potencial como fonte de linhagens (COCKERHAM, 1983). Mesmo assim, é possível avaliar populações, de modo aproximado, para que se tenha uma visão das suas potencialidades. A primeira maneira é conhecer a capacidade geral de combinação, outra é a que se baseia na depressão por endogamia, quando submetida à autofecundação (VENCOVSKY \& BARRIGA, 1992). Com base nos resultados das estimativas das médias esperadas nas linhagens pode-se inferir que as populações BRS-ANGELA e SC 002 apresentam maior probabilidade de sucesso na obtenção de linhas homozigóticas de boa capacidade de expansão. A população BRS-ANGELA deve ser utilizada para extrair linhagens que combinam capacidade de expansão e rendimento.

\section{AGRADECIMENTOS}

À Professora Dr ${ }^{\mathrm{a}}$ Valéria Carpentieri Pípolo, do Departamento de Agronomia da Universidade Estadual de Londrina-PR, pela gentileza em ceder as populações utilizadas neste trabalho.

Ao Conselho Nacional de Desenvolvimento Científico e Tecnológico (CNPq), pela concessão de bolsa ao pesquisador Scapim.

\section{REFERÊNCIAS}

BRUNSON, A.M. Popcorn breeding. In: U.S. Department Agricultural. Year book of Agriculture. Washington D.C., 1937. p.395-404.

COCKERHAM, C.C. Covariances of relatives from selffertilization. Crop Science, Madison, v.23, p.1177-1180, 1983.

DOFING, S.M. et al. A inheritance of expansion volume and yield in two popcorn x dent crosses. Crop Science, Madison, v.31, p.715-718, 1991.
GALVÃO, J.C.C. et al. Comportamento de híbridos de milhopipoca em Coimbra, Minas Gerais. Ceres, Viçosa, v.47, p.201$218,2000$.

GARDNER C.O. Teoria de genética estadistica aplicable as las medias de variedades, sus cruces y poblaciones afines. Fitotecnica Latinoamericana, Caracas, v.2, p.11-22, 1965.

GOOD, R.L.; HALLAUER, A.R. Inbreeding depression in maize by selfing and full-sibbing. Crop Science, Madison, v.17, p.935-940, 1977.

GOMEZ, A.K.; GOMEZ, A.A. Statistical procedures for agricultural research. New York: J. Wiley, 1984. 680p.

HALLAUER, A.R.; SEARS, J.H. Changes in quantitative traits associated with inbreeding in a synthetic variety of maize. Crop Science, Madison, v.13, p.327-330, 1973.

HALLAUER, A.R.; MIRANDA FILHO, J.B. Quantitative genetics in maize breeding. Ames: Iowa State University, 1981. 468p.

JONES, D.F. Continued inbreeding in maize. Genetics, Bethesda, v.24, p.462-473, 1939.

LARISH, L.L.B.; BREWBAKER, J.L. Diallel analyses of temperate and tropical popcorns. Maydica, Bergamo, v.44, p.279-384, 1999.

LIMA, M. et al. Inbreeding depression in Brazilian populations of maize (Zea mays L.). Maydica, Bergamo, v.29, p.203-215, 1984.

NASS, L.L.; MIRANDA FILHO, J.B. Inbreeding depression rates of semi-exotic maize (Zea mays L) populations. Revista Brasileira de Genética, Ribeirão Preto, v.18, p.585-592, 1995.

PACHECO, C.A.P. et al. Estimativas de parâmetros genéticos nas populações CMS-42 e CMS-43 de milho-pipoca. Pesquisa Agropecuária Brasileira, Brasília, v.33, n.12, p.995-2001, 1998.

PACHECO, C.A .P. et al. BRS ANGELA - variedade de milhopipoca. Sete Lagoas: EMBRAPA-Milho e Sorgo, 2001. 6p.

PEREIRA, M.G.; AMARAL JÚNIOR, A.T. Estimation of genetic components in popcorn based on the nested design. Crop Breeding and Applied Biotechnology, Londrina, v.1, n.1, p.3-10, 2001.

SAWAZAKI, E. Melhoramento do milho-pipoca. Campinas: Instituto Agronômico de Campinas, 1995. 21p.

SAWAZAKI, E. et al. Potencial de linhagens locais de milhopipoca para síntese de híbridos. Bragantia, Campinas, v.59, n.2, p.143-151, 2000.

SAWAZAKI, E. A cultura do milho-pipoca no Brasil. O Agronômico, Campinas, v.53, n.2, p.11-13, 2001.

SIMON, G.A. et al. Depressão por endogamia em populações de milho-pipoca. Bragantia, Campinas, v.63, n.1, p.55-62, 2004.

SCAPIM, C.A. et al. Análise dialélica e heterose de populações de milho-pipoca. Bragantia, Campinas, v.61, n.3, p.219230, 2002. 
SCAPIM, C.A. et al. Uma proposta de classificação dos coeficientes de variação para cultura do milho. Pesquisa Agropecuária Brasileira, Brasília, v.30, n.5, p.683-686, 1995.

VASAL, S.K. et al. Effect of S3 recurrent selection in four tropical maize populations on their selfed and randomly mated generations. Crop Science, Madison, v.35, p.697-702, 1995.

VENCOVSKY, R.; BARRIGA, P. Genética biométrica no fitomelhoramento. Ribeirão Preto: Sociedade Brasileira de Genética, 1992. 496p.
VENDRUSCOLO, E.C.G. Comparação de métodos e avaliação da adaptabilidade e estabilidade de genótipos de milho-pipoca (Zea mays L.) na região Centro-Sul do Brasil. 1997. 79f. Dissertação (Mestrado em Agronomia) - Curso de Pós-graduação em Agronomia, Universidade Estadual de Maringá.

VIANNA, R.T. et al. Inbreeding depression of several introduced populations of maize (Zea mays L.). Maydica, Bergamo, v.27, p.151-157, 1982

ZINSLY, J.R.; MACHADO, J. Milho-pipoca. In: PATERNIANI, E.; VIÉGAS, G.P. (eds). Melhoramento e produção de milho. 2.ed. Campinas: Fundação Cargill, 1987. p.431-421. 